\title{
Tunable Spin-Orbit Coupling via Strong Driving in Ultracold-Atom Systems
}

\author{
K. Jiménez-García, ${ }^{1,2}$ L. J. LeBlanc, ${ }^{1}$ R. A. Williams, ${ }^{1}$ M. C. Beeler, ${ }^{1}$ C. Qu,${ }^{3}$ M. Gong, ${ }^{3}$ C. Zhang, ${ }^{3}$ and I. B. Spielman ${ }^{1, *}$ \\ ${ }^{1}$ Joint Quantum Institute, National Institute of Standards and Technology, \\ and University of Maryland, Gaithersburg, Maryland 20899, USA \\ ${ }^{2}$ Departamento de Física, Centro de Investigación y Estudios Avanzados del Instituto Politécnico Nacional, \\ México Distrito Federal 07360, México \\ ${ }^{3}$ Department of Physics, the University of Texas at Dallas, Richardson, Texas 75080 USA
}

(Received 12 December 2014; published 24 March 2015)

\begin{abstract}
Spin-orbit coupling is an essential ingredient in topological materials, conventional and quantum-gasbased alike. Engineered spin-orbit coupling in ultracold-atom systems-unique in their experimental control and measurement opportunities-provides a major opportunity to investigate and understand topological phenomena. Here we experimentally demonstrate and theoretically analyze a technique for controlling spin-orbit coupling in a two-component Bose-Einstein condensate using amplitude-modulated Raman coupling.
\end{abstract}

DOI: 10.1103/PhysRevLett.114.125301

PACS numbers: 67.85.-d, 32.10.Fn, 33.60.+q, 37.10.Gh

The properties of electronic materials are deeply entwined with their band structure-or, more generally, their single-particle spectrum-which gives rise to conductors, semiconductors, conventional insulators, and now topological insulators [1]. Understanding and controlling band structure in new ways, therefore, allows access to new phenomena. Spin-orbit coupling (SOC) plays a fundamental role in most topological materials, linking the spin and the momentum of quantum particles. The introduction of time-periodic perturbations to topologically trivial systems (quantum wells, solid-state materials, and ultracold atoms) can drive phase transitions to new "Floquet topological phases" $[2,3]$. For example, Floquet topological insulators arise from topologically trivial materials with spin-orbit coupling through time-periodic perturbations [2].

In such materials, topological properties are induced and controlled by periodically modulating various terms in the single-particle Hamiltonian. In ultracold-atom systems, we precisely design, introduce, and manipulate SOC by coupling the internal atomic degrees of freedom with laser fields [4]. Here, we illuminated an ultracold-atom system with a pair of "Raman" lasers, inducing SOC in an effective twolevel system [5-10] with SOC strength defined by the laser geometry alone. In this Letter, we experimentally show that strongly modulating the Raman coupling tunes the SOC, independently of the geometry and in agreement with theory.

We engineered SOC in an effective two-level atom in a uniform magnetic field $B \hat{\mathbf{e}}_{z}$ that Zeeman split the energy levels by $\hbar \omega_{Z}=g_{F} \mu_{B} B$, where $\mu_{B}$ is Bohr's magneton and $g_{F}$ is the Landé $g$ factor. These levels were coupled by a pair

Published by the American Physical Society under the terms of the Creative Commons Attribution 3.0 License. Further distribution of this work must maintain attribution to the author(s) and the published article's title, journal citation, and DOI. of orthogonally polarized Raman laser beams with angular frequencies $\omega_{L}$ and $\omega_{L}+\Delta \omega$ and a relative phase, as shown in Fig. 1. The lasers' frequency difference $\Delta \omega$ was set near $\omega_{Z}$, naturally defining an experimentally tunable detuning $\delta_{0}=\Delta \omega-\omega_{Z}$.

In our experiment, we selected as our two-level system, the $\left|m_{F}=0,-1\right\rangle \equiv|\uparrow, \downarrow\rangle$ hyperfine states of the $5 S_{1 / 2}$, $f=1$ manifold of ${ }^{87} \mathrm{Rb}$ [5]. The Raman laser field coupled spin states $\left|\downarrow, q_{x}=k_{x}-k_{L}\right\rangle$ to $\left|\uparrow, q_{x}=k_{x}+k_{L}\right\rangle$ differing in momentum by $2 k_{L}$, where $q_{x}=k_{x} \pm k_{L}$ denotes the quasimomentum. The recoil momentum $k_{L}=2 \pi \sin (\theta / 2) / \lambda$ and energy $E_{L}=\hbar^{2} k_{L}^{2} / 2 m$ set the relevant momentum and energy scales for Raman lasers intersecting at an angle $\theta$; here, $\lambda$ is the laser wavelength, and $m$ is the atomic mass. In this experiment, $\theta=\pi / 2$, as shown in Fig. 1 [12].

In the frame rotating at angular frequency $\Delta \omega$ and after making the rotating wave approximation, the Hamiltonian combining both the kinetic and Raman coupling contributions is [5]

$$
\hat{H}=\left(\frac{\hbar^{2} q_{x}^{2}}{2 m}+E_{L}\right) \hat{1}+\frac{\hbar \Omega}{2} \hat{\sigma}_{x}+\frac{\hbar \delta_{0}}{2} \hat{\sigma}_{z}+\alpha_{0} q_{x} \hat{\sigma}_{z},
$$

where $\Omega \propto E_{A}^{*} E_{B}$ is the Raman coupling strength, $\hat{\sigma}_{x, y, z}$ are the Pauli matrices, and $E_{A, B}$ are the complex-valued optical electric field strengths [Fig. 1(a)]. The last term describes SOC - an equal sum of Rashba and Dresselhaus SOCwith strength $\alpha_{0}=2 E_{L} / k_{L} \propto k_{L}$. The resulting energy bands of the laser dressed atomic system $E_{ \pm}\left(q_{x}\right)$ are obtained by diagonalizing $\hat{H}$ as a function of the quasimomentum $q_{x}$. We focused on the atoms in the lowest energy band, where they experienced the energy-momentum dispersion relation given by $E_{-}\left(q_{x}\right)$.

The SOC strength $\alpha_{0} \propto \sin (\theta / 2)$ depends only on the momentum difference between the Raman laser beams and 
(a)

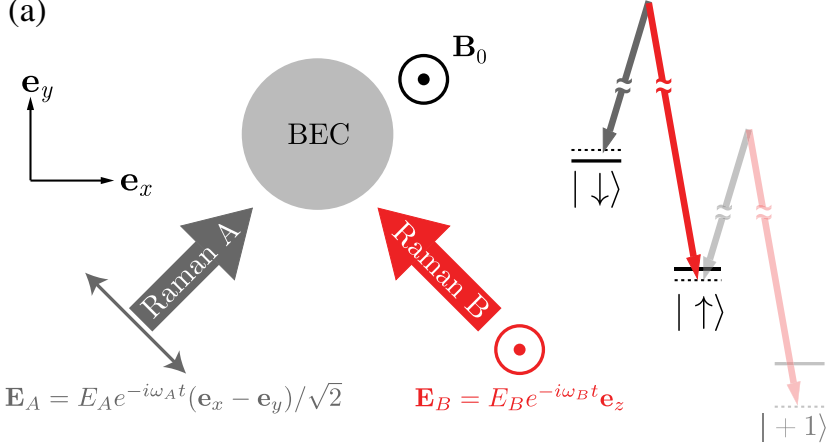

(b)

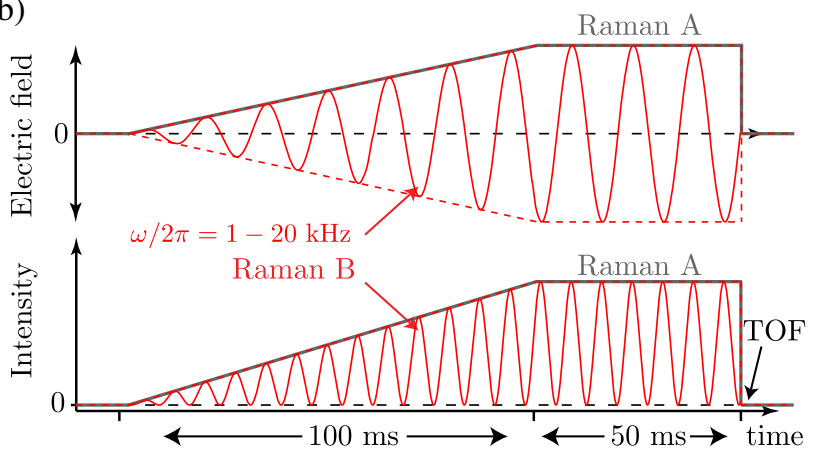

(c)

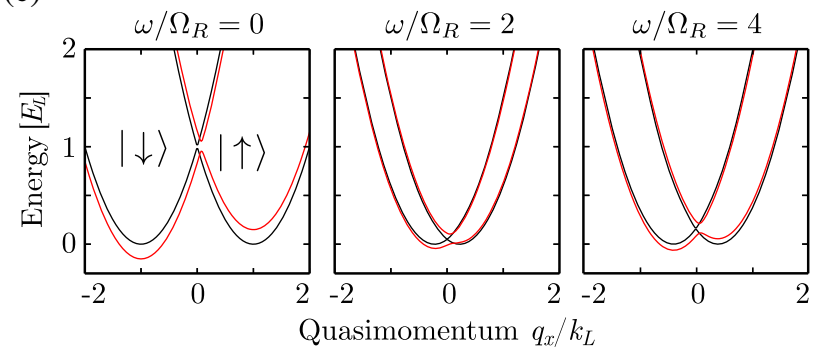

FIG. 1 (color online). Setup and level diagram. (a) A uniform bias field $B \mathbf{e}_{z}$ Zeeman splits the hyperfine sublevels of an $f=1$ ${ }^{87} \mathrm{Rb} \mathrm{BEC}$, and a pair of Raman beams illuminates the atoms. The field $B$ generates a large quadratic Zeeman shift $\hbar \epsilon / E_{L} \gg 1$, which effectively decouples the third spin state. By adjusting the detuning, we select the states $|-1\rangle=|\downarrow\rangle$ and $|0\rangle=|\uparrow\rangle$ to form an effective two-level system. (b) Schematic of electric field and associated intensity ramps used in the experiment [11] to modulate the Raman coupling strength $\Omega(t)$. (c) Calculated dispersion relations from the time-periodic single-particle Hamiltonian. Black curves indicate $\delta=0$ and $\Omega_{0}=0$, while red curves indicate $\delta=-0.3 E_{L}$ and $\Omega_{0}=0.1 E_{L}$.

reaches its maximum for counterpropagating beams, $\theta=\pi$. Here we demonstrate a method for tuning the SOC strength $\alpha_{0}$ in real time: modulating the coupling strength $\hbar \Omega$ by controlling the intensity and phase of the Raman lasers [11,13-15]. For rapid drive of the form $\Omega(t)=$ $\Omega_{0}+\Omega_{R} \cos (\omega t)$ and $\hbar \omega \gg 4 E_{L}$, the effective Floquet Hamiltonian retains the form of Eq. (1) but with renormalized coefficients $\Omega=\Omega_{0}, \quad \delta=J_{0}\left(\Omega_{R} / \omega\right) \delta_{0}$, and $\alpha=J_{0}\left(\Omega_{R} / \omega\right) \alpha_{0} . J_{0}$ is the zeroth order Bessel function of the first kind; i.e., $\alpha$ is an oscillatory function of $\Omega_{0} / \omega$, generally decreasing in amplitude as $\Omega_{0} / \omega$ increases [13].
Our experiments started with nearly pure ${ }^{87} \mathrm{Rb}$ BECs in a crossed optical dipole trap, with frequencies $\left(f_{x}, f_{y}, f_{z}\right)=(32,37,100) \mathrm{Hz}$. Prior to dressing the atoms with the Raman lasers, we prepared these BECs either in the spin state $|\downarrow\rangle,|\uparrow\rangle$ or an equal superposition thereof. The $B=2.142 \mathrm{mT}$ bias field Zeeman split the $|\uparrow\rangle$ and $|\downarrow\rangle$ states by $\omega_{Z} / 2 \pi \approx 15 \mathrm{MHz}$, detuning the unwanted $\left|m_{F}=+1\right\rangle$ state by $36 E_{L}$ from resonance.

We optically dressed the atoms with a pair of $\lambda=790.1 \mathrm{~nm}$ Raman lasers propagating along $\mathbf{e}_{y} \pm \mathbf{e}_{x}$ (Fig. 1) and controlled $\delta_{0}$ by making small changes to $B$. The Raman coupling strength $\hbar \Omega$ was experimentally controlled by the intensity of the lasers, and we inverted the sign of $\Omega$ by shifting the beams' relative phase by $\pi$ [11]. Each Raman beam (labeled $A$ and $B$, respectively) was ramped from zero to its final intensity in $100 \mathrm{~ms}$ following a linear envelope; however, the intensity of Raman laser $B$ was additionally modulated sinusoidally [Fig. 1(b)]. The atoms were then held for $50 \mathrm{~ms}$, after which all potentials were turned off. The atomic ensemble expanded for a $34.45 \mathrm{~ms}$ time of flight (TOF) before absorption imaging. Using a magnetic field gradient during part of the TOF, we separated the spin components along $\mathbf{e}_{y}$.

We determined the SOC strength from direct measurements of atomic momentum distributions as shown in Fig. 2. We first studied systems driven at $\omega / 2 \pi=$ $10 \mathrm{kHz}$ and $20 \mathrm{kHz}$ and found momentum distributions in excellent agreement with the expected behavior; i.e., the atoms adiabatically followed degenerate ground states of the driven Raman Hamiltonian located at $q_{x}= \pm\left(\alpha / \alpha_{0}\right) k_{L}$ as we tuned $\Omega_{R} / \omega$. Figure 3(a) constitutes the main result of our work and demonstrates experimental control on the SOC strength $\alpha\left(\Omega_{R} / \omega\right)$ for systems driven at $\omega / 2 \pi=$ $10 \mathrm{kHz}$. As $\Omega_{R} / \omega$ increased, we observed the Besselfunction behavior of $\alpha$.

We compared our data to the simulated dynamics of the BEC governed by the time-dependent Gross-Pitaevskii equation (TDGPE)

$$
i \hbar \frac{\partial \Psi}{\partial t}=\left[H(t)+V(\mathbf{r})+H_{I}\right] \Psi
$$

where $\Psi=\left(\Psi_{\downarrow}, \Psi_{\uparrow}\right)^{T}$ is a two-component wave function, $H(t)$ is the coupling Hamiltonian of Eq. (1), $V(\mathbf{r})$ corresponds to a harmonic confining potential, and $H_{I}$ describes atomic density-density interactions. We numerically simulated BECs with $N=10^{5}$ atoms and obtained the $t=0$ initial state (before modulation) using imaginary time evolution with either the BEC initially polarized as $|\downarrow\rangle$ at $q_{x}=-1\left(\hbar \delta_{0}=-0.5 E_{L}\right)$ or as a balanced spin superposition at $q_{x}= \pm 1\left(\hbar \delta_{0}=0\right)$. We then explicitly time evolved with the TDGPE [16] including the full timedependent Raman coupling $\Omega(t)=\Omega_{0}+\Omega \cos (\omega t)$, where $\hbar \Omega_{0}=0.3 E_{L}$ is the small constant offset, and $\Omega$ is slowly ramped on as in the experimental procedure [17]. 
(a)
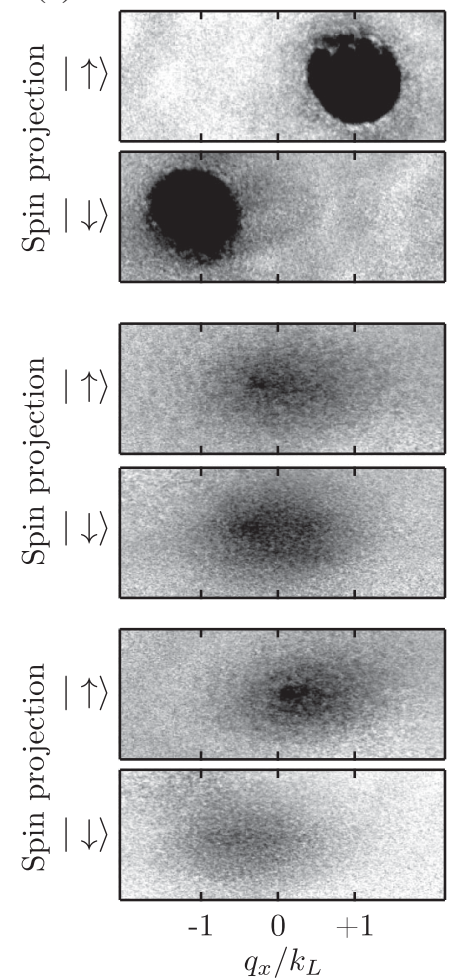

(b)
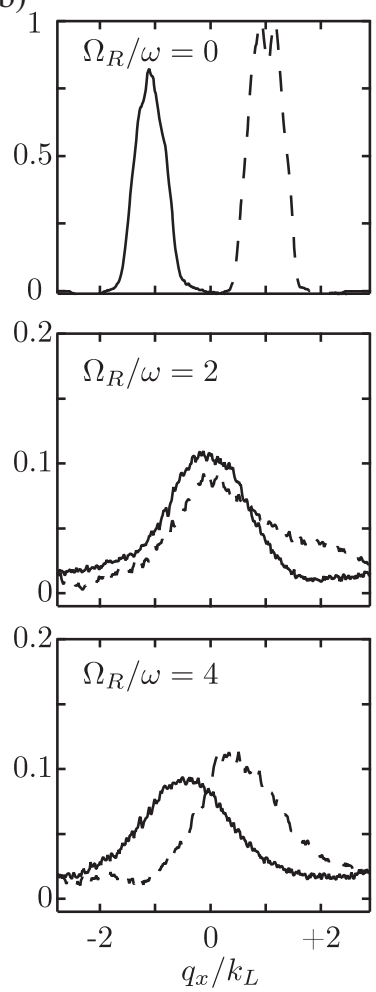

FIG. 2. Absorption imaged TOF density distributions for $\omega / 2 \pi=10 \mathrm{kHz}$. The top, middle, and bottom panels correspond to $\Omega_{R} / \omega=0,2$, and 4 , respectively. (a) (Top) When $\Omega_{R} / \omega=0$, the spin states are maximally separated by $\Delta k_{x}=2 k_{L}$. (Middle) When $\Omega_{R} / \omega=2$, the quasimomentum separation is practically zero. (Bottom) When $\Omega_{R} / \omega=4$, the spin states again separate in quasimomentum. (b) The continuous (dashed) lines correspond to the optical depth integrated along $\mathbf{e}_{y}$, for $|\downarrow\rangle(|\uparrow\rangle)$.

Figure 3(b) displays the calculated density distributions $\left|\psi\left(q_{x}\right)\right|^{2}=\iint\left|\psi\left(q_{x}, q_{y}, q_{z}\right)\right|^{2} d q_{y} d q_{z}$ as a function of time for both the balanced spin superposition and the spin polarized initial states. We determined the final quasimomentum by finding the center of small dipole oscillations which occur around the band minimum. In both cases, oscillations of the BEC's quasimomentum around the local band minimum (for $t>100 \mathrm{~ms}$ ) result from imperfect adiabaticity during the ramp-up process of the Raman lasers. In the experiment, we found that the system relaxed very rapidly to the local band minimum giving the measured quasimomentum shown in Fig. 3(a).

Furthermore, we experimentally observed that as $J_{0}\left(\Omega_{R} / \omega\right)$ became negative, the individual spins did not pass through $q_{x}=0$ as might be expected but rather "reflected" and continued following the Bessel envelope without changing sign [Fig. 3(a)]; this was the case for both the spin superposition and the single-spin data. Our simulations show that this reflection is present when the Raman coupling offset $\hbar \Omega_{0}$ and detuning $\hbar \delta_{0}$ are small but nonzero, as described below.

(a)
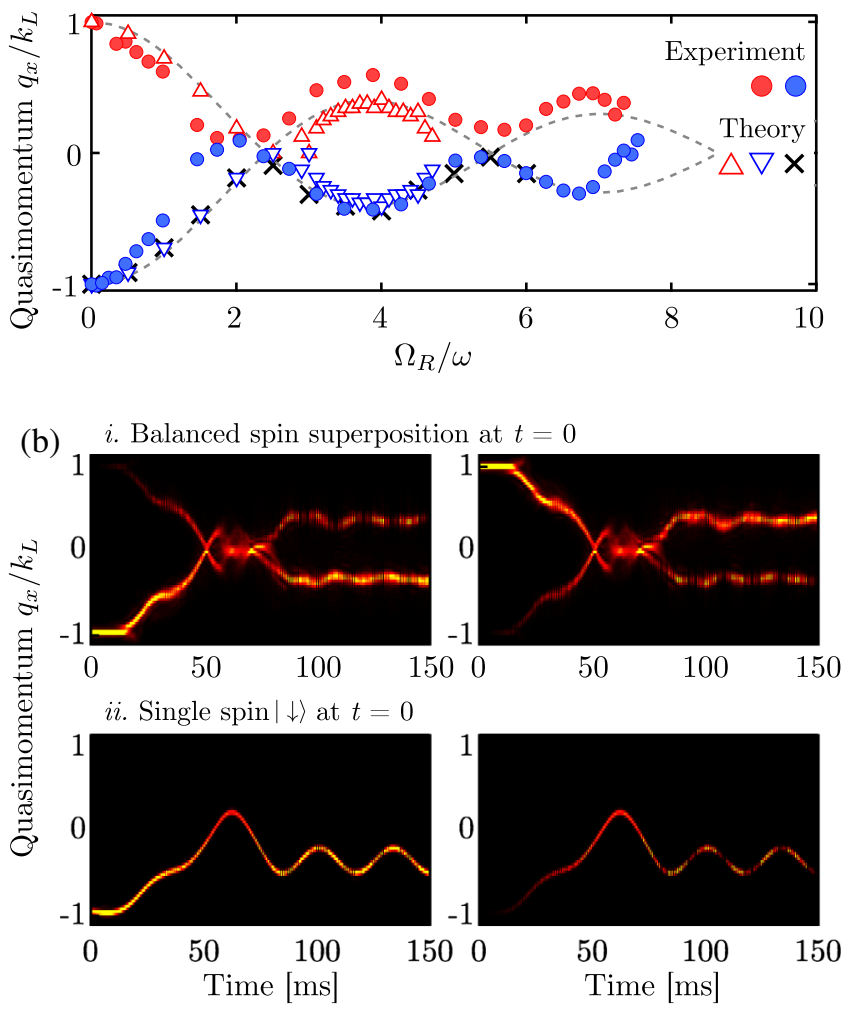

FIG. 3 (color online). Tunable SOC strength. (a) Quasimomentum as a function of $\Omega_{R} / \omega$. Closed (open and crossed) symbols correspond to measurements (calculations) of systems driven at $\omega / 2 \pi=10 \mathrm{kHz}(20 \mathrm{kHz})$ for an initial balanced spin superposition (for initial spin superposition and spin polarized states, respectively). The blue (red) symbols correspond to $|\downarrow\rangle(|\uparrow\rangle)$ atoms and the dashed curves correspond to $J_{0}\left(\Omega_{R} / \omega\right)$ (b) Time evolution of the density distributions in momentum space for $\Omega_{R} / \omega=4$ with relevant experimental initial states. In both calculations, a reflection of the quasimomentum occurs at $q_{x}=0$ for constant $\hbar \Omega_{0}=0.3 E_{L}$ and $\omega / 2 \pi=20 \mathrm{kHz}$ [see panel (a)]. For the single-spin case, we used a finite detuning $\hbar \delta_{0}=-0.5 E_{L}$ [17]. (bi) Balanced spin superposition at $t=0$, (bii) Single spin $|\downarrow\rangle$ at $t=0$.

For the initial balanced spin superposition, the states $\left|\downarrow, q_{x}=-1\right\rangle$ and $\left|\uparrow, q_{x}=1\right\rangle$ move close to each other under fast modulation [Fig. 3(bi)]. As time evolves, each state develops a dressed state partner: this additional state is negligible for small $\Omega(t) / \omega$ (short time); however, it becomes more relevant for large $\Omega(t) / \omega$ (long time). By averaging over a short time period (longer than the spin flip time), we see the main spin components cross $q_{x}=0$ and flip their spin giving rise to the observation of a spin reflection in the experiment [Fig. 3(a)].

For the initial spin polarized state $|\downarrow\rangle$, the calculated time evolution with $\delta_{0}=0$ shows that the $|\downarrow\rangle$ component will cross $q_{x}=0$ and be converted to $|\uparrow\rangle$; however, when $\delta_{0} \neq 0$, we observe a reflection in quasimomentum since the global minimum of the band structure stays at the same 
side. Figure 3(bii) shows the time evolution of the single spin with an additional small Raman coupling offset $\hbar \Omega_{0} \sim 0.3 E_{L}$ [17].

Physically, when these terms are small, the atoms undergo a Majorana spin flip as $J_{0}\left(\Omega_{R} / \omega\right)$ changes sign but are able to adiabatically follow when they are nonzero. Because $\alpha, \delta \propto J_{0}\left(\Omega_{R} / \omega\right)$ change sign simultaneously, the $q_{x}$ 's for the local minima in $E_{-}\left(q_{x}\right)$ associated with each spin state do not change sign, so the local minima reflect from $q_{x}=0$. This argument can also be understood by considering the red curves in Fig. 1(c) showing a progression of effective SOC dispersion relations with nonzero $\Omega_{0}$ and $\delta$; it is evident that atoms which start in the lower (left) minimum will stay in that minimum even after the minima have merged and separated once more.

In the experiment, $\Omega(t)$ was determined by the intensity and relative phase of two Raman lasers as controlled by acousto-optic modulators (AOMs); it is likely that a small dc contribution to the AOM's drive gave $\Omega(t)$ a small nonzero average at the $5 \%$ level. In the strong driving region, $\hbar \Omega_{R}>\hbar \omega \approx 10 E_{L}$, this corresponds to a $\hbar \Omega_{0} \sim 0.5 E_{L}$ offset. Furthermore, in our experiment, small detunings $\hbar\left|\delta_{0}\right| \sim 0.1 E_{L}$ were generally present.

In addition, Fig. 2 shows that the system is heated in the presence of the drive. Figure 4(a) parametrizes this effect in terms of the temperature of the driven system as a function of driving frequency at fixed $\Omega_{R} / \omega=2.9$. The heating was most pronounced in the range $2 \mathrm{kHz}<\omega / 2 \pi<7 \mathrm{kHz}$ and reached a plateau for $\omega / 2 \pi>10 \mathrm{kHz}$. Because our atoms are continuously evaporating from the shallow optical dipole trap, this heating drives rapid atom loss, as plotted in Fig. 4(b) for $\Omega_{R} / \omega=4.5$ and $\omega / 2 \pi=10 \mathrm{kHz}$. This unwanted heating is present as dephasing in our zerotemperature GPE model and described in terms of the BEC's stability under driving.

Stability depends on the modulation frequency: for very large $\omega$, the time-dependent terms average out (rotating wave approximation), and the dynamics of the BEC follow a stable effective modulation; for very small $\omega$, the SOC strength barely changes and the system is also stable. In the intermediate regime, we use Floquet theory to describe the strong instability of the modulated BEC [17]. Figure 4(c) shows the Floquet band structure of a minimal model spanned by the basis $\{|\downarrow, n=0\rangle,|\uparrow, n=+1\rangle,|\uparrow, n=-1\rangle\}$, where $|\sigma, n\rangle$ denotes the state with spin $\sigma$ and Floquet sideband index $n$. We consider a BEC in the initial state $\left|\downarrow, q_{x}=-1\right\rangle$ green point in [Fig. 4(c)]. At a critical driving frequency $\hbar \omega \sim 4 E_{L} \sim h \times 7.36 \mathrm{kHz}$, a gap opens and the BEC is suddenly split into two bands. The component in the lower band is not stable because of its associated negative effective mass and since strong nonlinear interactions lead to complicated collective excitations. When $\hbar \omega \lesssim 4 E_{L}$, the majority of atoms populate the lower band and may experience an unstable point after a short time of dipole motion; therefore, the BEC is not stable and the
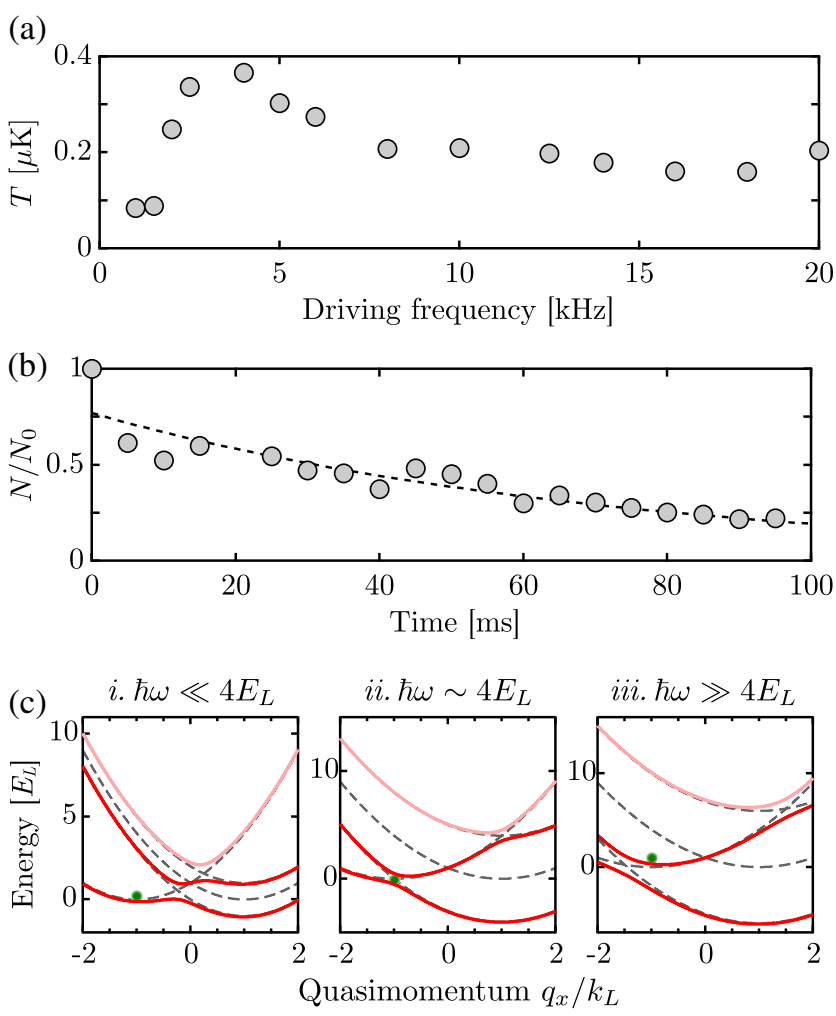

FIG. 4 (color online). Heating in the optically driven SOC system. (a) Temperature of the atomic sample measured from the thermal fraction of the momentum distribution as a function of $\omega$ at $\Omega_{R} / \omega=2.9$. (b) Number of atoms remaining in the driven system for $\Omega_{R} / \omega=4.5$, an exponential fit gave a $1 / e$ lifetime $\tau=72.3 \mathrm{~ms}$. (c) Floquet band structure at fixed coupling $\Omega_{R}$ for driving frequencies $\omega / 2 \pi=1,7.36 \mathrm{kHz}$ and $20 \mathrm{kHz}$. (ci) $\hbar \omega \ll 4 E_{L}$, (cii) $\hbar \omega \sim 4 E_{L}$, and (ciii) $\hbar \omega \gg 4 E_{L}$.

heating is strongest at $\hbar \omega \sim \mathrm{h} \times 5 \mathrm{kHz}$. For $\hbar \omega \ll 4 E_{L}$, the $\mathrm{BEC}$ is stable as it follows the lower band which now exhibits a local minimum. For $\hbar \omega \gg 4 E_{L}$, the BEC adiabatically follows the upper band minimum and is stable.

The unstable range of drive frequencies is larger for stronger interactions and vanishes for vanishing interactions where the simple effective quasieigenstates become exact. In the unstable region, the BEC is destroyed after a modulation time of just $10 \mathrm{~ms}$ to $50 \mathrm{~ms}$, at which point the momentum space distribution is dominated by high momentum excitations, which would be interpreted as thermal excitations observed in the experiment. In general, the exact size of the unstable region depends on both $\Omega_{R} / \omega$ and interaction strength; thus, while the experimental data fall into the unstable region, the exact location of the boundary may be influenced by the constant atom number $\left(N=10^{5}\right)$ used in the numerical simulation. For this experiment, we avoided the larger $\omega$ 's required to enter the stable regime because this also requires larger $\Omega_{R}$, leading to unwanted spontaneous-emission-driven heating (not included in our GPE calculation). 
SOC is a critical ingredient in the generation and understanding of important condensed matter phenomena, and achieving real-time control of its coupling strength represents a step forward in the quest for novel quantum matter. Tunable SOC provides a powerful tool for investigating important open problems in quantum many-body physics, such as the properties of topological phases of matter. Here, we demonstrated a technique to control the coupling strength in a light-induced SOC system. Our technique relied on modulating the Raman laser field illuminating an ultracold-atom system. The measured SOC strength as a function of the dimensionless Raman coupling strength $\Omega_{R} / \omega$ was in good agreement with theory. This work showed that Raman modulation is a powerful way to control SOC in quantum gases, in analogy to modulated lattice experiments $[15,18]$.

We appreciate enlightening conversations with G. Juzeliunas, N. R. Cooper, and W. D. Phillips; additionally, we thank Dina Genkina for carefully reading our manuscript. This work was partially supported by the ARO with funding from DARPA's OLE program and the AtomtronicsMURI and the NSF through the JQI Physics Frontier Center. K. J.-G. thanks CONACYT; L. J. L. thanks NSERC; M. C. B. thanks the NIST-ARRA program, and C. Q., M. G., and C.Z. are supported by ARO (Grant No. W911NF-12-10334), AFOSR (Grant No. FA9550-13-1-0045), and NSF-PHY (Grant No. 1249293).

*ian.spielman@nist.gov

[1] M. Z. Hasan and C. L. Kane, Rev. Mod. Phys. 82, 3045 (2010).

[2] N. H. Linder, G. Refael, and V. Galitski, Nat. Phys. 7, 490 (2011).
[3] G. Jotzu, M. Messer, R. Desbuquois, M. Lebrat, T. Uehlinger, D. Greif, and T. Esslinger, Nature (London) 515, 237 (2014).

[4] J. Dalibard, F. Gerbier, G. Juzeliūnas, and P. Öhberg, Rev. Mod. Phys. 83, 1523 (2011).

[5] Y.-J. Lin, K. Jiménez-García, and I. B. Spielman, Nature (London) 471, 83 (2011).

[6] I. B. Spielman, Phys. Rev. A 79, 063613 (2009).

[7] L. W. Cheuk, A. T. Sommer, Z. Hadzibabic, T. Yefsah, W. S. Bakr, and M. W. Zwierlein, Phys. Rev. Lett. 109, 095302 (2012).

[8] P. Wang, Z.-Q. Yu, Z. Fu, J. Miao, L. Huang, S. Chai, H. Zhai, and J. Zhang, Phys. Rev. Lett. 109, 095301 (2012).

[9] J.-Y. Zhang, S.-C. Ji, Z. Chen, L. Zhang, Z.-D. Du, B. Yan, G.-S. Pan, B. Zhao, Y.-J. Deng, H. Zhai, S. Chen, and J.-W. Pan, Phys. Rev. Lett. 109, 115301 (2012).

[10] C. Hamner, C. Qu, Y. Zhang, J. Chang, M. Gong, C. Zhang, and P. Engels, Nat. Commun. 5, 4023 (2014).

[11] G. Juzeliūnas and I. Spielman, New J. Phys. 14, 123022 (2012).

[12] For this effective two-level system, we redefine the detuning as $\delta=\delta^{\prime}+\epsilon$ to account for the quadratic contribution to the Zeeman shift, $\epsilon$ [5].

[13] Y. Zhang, G. Chen, and C. Zhang, Sci. Rep. 3, 1937 (2013).

[14] B. Dóra, J. Cayssol, F. Simon, and R. Moessner, Phys. Rev. Lett. 108, 056602 (2012).

[15] J. Struck, C. Ölschläger, M. Weinberg, P. Hauke, J. Simonet, A. Eckardt, M. Lewenstein, K. Sengstock, and P. Windpassinger, Phys. Rev. Lett. 108, 225304 (2012).

[16] W. Bao, D. Jaksch, and P. A. Markowich, J. Comput. Phys. 187, 318 (2003).

[17] See the Supplemental Material at http://link.aps.org/ supplemental/10.1103/PhysRevLett.114.125301 for details on the numerical model and a discussion of heating.

[18] H. Lignier, C. Sias, D. Ciampini, Y. Singh, A. Zenesini, O. Morsch, and E. Arimondo, Phys. Rev. Lett. 99, 220403 (2007). 